\title{
MicroRNA-34a-5p enhances sensitivity to chemotherapy by targeting AXL in hepatocellular carcinoma MHCC-97L cells
}

\author{
XIAO-YUN LI ${ }^{1 *}$, JING-YUN WEN $^{1 *}$, CHANG-CHANG JIA ${ }^{2 *}$, TIAN-TIAN WANG ${ }^{1}$, XING LI $^{1}$, MIN DONG $^{1}$, \\ QU LIN $^{1}$, ZHAN-HONG CHEN ${ }^{1}$, XIAO-KUN MA ${ }^{1}$, LI WEI ${ }^{1}$, ZE-XIAO LIN ${ }^{1}$, DAN-YUN RUAN ${ }^{1}$, JIE CHEN ${ }^{1}$, \\ DONG-HAO WU ${ }^{1}$, WEI LIU ${ }^{2}$, YAN TAI ${ }^{2}$, ZHI-YONG XIONG $^{2}$, XIANG-YUAN WU ${ }^{1}$ and QI ZHANG ${ }^{2}$ \\ ${ }^{1}$ Department of Medical Oncology, The Third Affiliated Hospital of Sun Yat-sen University; \\ ${ }^{2}$ Guangdong Provincial Key Laboratory of Liver Disease Research, Sun Yat-sen University, \\ Guangzhou, Guangdong 510630, P.R. China
}

Received July 18, 2014; Accepted April 14, 2015

DOI: 10.3892/ol.2015.3654

\begin{abstract}
Mature microRNA (miRNA) 34a-5p, which is a well-known tumor suppressor in hepatitis virus-associated hepatocellular carcinoma (HCC), plays an important role in cell processes, such as cell proliferation and apoptosis, and is therefore an optimal biomarker for future clinical use. However, the role of miRNA-34a-5p in chemoresistance has yet to be identified. In the present study, the expression of miRNA-34a-5p was assessed by an in situ hybridization assay in HCC tissues and was found to be significantly decreased compared with the pericarcinomatous areas of the tissue specimens, which consisted of samples obtained from 114 patients with HCC. High expression of miRNA-34a-5p was found to be associated with a favorable overall survival time in HCC patients. Functional tests performed by transfecting miRNA-34a-5p mimics or inhibitors into MHCC-97L cells illustrated that miRNA-34a-5p inhibited proliferation, elevated apoptosis and decreased chemoresistance to cisplatin in HCC cells. AXL is the direct target of miRNA-34a-5p, as confirmed by sequence analysis and luciferase assay. Transfection of the cells with small interfering RNA for AXL (siAXL) increased the apoptosis ratio of the MHCC-97L cell line. Transfection with siAXL led to similar biological behaviors in the MHCC-97L cells to those induced by ectopic expression
\end{abstract}

Correspondence to: Mr. Xiang-Yuan Wu, Department of Medical Oncology, The Third Affiliated Hospital of Sun Yat-sen University, 600 Tianhe Road, Guangzhou, Guangdong 510630, P.R. China

E-mail:wuxiangy@mail.sysu.edu.cn

Ms. Qi Zhang, Guangdong Provincial Key Laboratory of Liver Disease Research, Sun Yat-sen University, 600 Tianhe Road, Guangzhou, Guangdong 510630, P.R. China

E-mail: kee_kee@126.com

${ }^{*}$ Contributed equally

Key words: microRNA34a-5p, cisplatin, chemoresistance, AXL, hepatocellular carcinoma of miRNA-34a-5p. Thus, it was concluded that miRNA-34a-5p enhanced the sensitivity of the cells to chemotherapy by targeting AXL in hepatocellular carcinoma. In addition, low expression of miRNA-34a-5p in HCC tissues yielded an unfavorable prognosis for patients with HCC that received radical surgery, due to the promotion of proliferation and an increase in chemoresistance in HCC cells.

\section{Introduction}

Hepatocellular carcinoma (HCC) is one of the most common malignant tumors worldwide and also demonstrates an increasing incidence and mortality, with a poor prognosis that limits the long-term survival rate of patients (1). In addition, the majority of patients are in the middle to late stage of disease at the time of diagnosis and have lost the opportunity for successful treatment (2). Although various treatments are improving, atypical clinical features and an intrinsic chemoresistance mechanism continue to limit the long-term survival of patients with HCC (3). Numerous efforts have been made to improve the sensitivity of HCC to chemotherapy. However, few attempts are successful (4) and the resistance mechanism requires additional study.

The optimal microRNA (miRNA) for clinical application is required to be involved in multiple behaviors in HCC development, such as cell proliferation, apoptosis and chemoresistance. The mature miRNA-34a-5p, which is well known as a tumor suppressor in hepatitis virus-associated HCC, plays an important role in cell processes like proliferation and apoptosis, which made it an optimal biomarker for future clinical usage (5-8). Low-expression miRNA-34a-5p was reported to be associated with certain characteristics and an unfavorable clinical outcome in a small number of studies $(9,10)$. In addition, miRNA-34a demonstrated an enhanced anti-proliferative ability by targeting c-met or B-cell lymphoma-2 (Bcl-2) in HCC cells $(9,10)$. However, the clinical importance of miRNA-34a-5p in processes such as chemoresistance has yet to be identified and the specific map of the mechanism associated with chemoresistance requires elucidation.

Therefore, the present study aimed to evaluate the significance of miRNA-34a-5p in patients with HCC that received 
radical surgery, and also aimed to identify the mechanism behind these effects, which include alterations in cell proliferation, apoptosis and chemoresistance. In addition, the current study attempted to identify the critical pathway and target gene of miRNA-34a-5p in patients with HCC.

\section{Materials and methods}

Patients. Between September 2009 and September 2012, the present study investigated a consecutive series of 114 HCC patients receiving radical surgery in the Cancer Center of Sun Yat-sen University (Guangzhou, Guangdong, China). The diagnosis of HCC was universally confirmed by pathology. In total, 114 patients were followed-up prior to mortality or to the most recent follow-up on October 30, 2013. The overall survival (OS) time was defined as the time from diagnosis to the date of death, or to the most recent follow-up if the patient remained alive. The progression-free survival (PFS) time was defined as the time between diagnosis and the date of local failure or distant metastasis, or the time between diagnosis and mortality or date of the most recent follow-up if progression did not occur. The present study was approved by the Clinical Ethics Review Boards of the Third Affiliated Hospital and Cancer Center of Sun Yat-sen University. Written informed consent was obtained from all patients at the time of admission.

Chemicals and reagents. Primary antibodies against AXL (rabbit anti-human monoclonal; cat. no. 4939; 1:1,000), c-Jun N-terminal protein kinase (JNK; Rabbit anti-human polyclonal; cat. no. 9252; 1:1,000), phosphorylated JNK (p-JNK; rabbit anti-human polyclonal; cat. no. 9251; 1:1,000), Bcl-2 (mouse anti-human monoclonal; cat. no. 15071; 1:1,000), phosphatidylinositol 3-kinase (PI3K; rabbit anti-human monoclonal; cat. no. 11889; 1:1,000), AKT, phosphorylated AKT (p-AKT; rabbit anti-human polyclonal; cat. no. 9272; 1:1,000), caspase-3 (rabbit anti-human monoclonal; cat. no. 9665; 1:1,000) and cleaved caspase-3 (rabbit anti-human monoclonal; cat. no. 9661; 1:1,000) were purchased from Cell Signaling Technology, Inc. (Danvers, MA, USA), and the $\beta$-actin antibody (mouse anti-human polyclonal; cat. no. sc-44478; 1:1,000) was purchased from Santa Cruz Biotechnology, Inc. (Dallas, TX, USA). The HRP-conjugated anti-rabbit IgG (cat. no. 7054, 1:5,000) secondary antibodies were purchased from Cell Signaling Technology, Inc. The cell-culture reagent Dulbecco's Modified Eagle's Medium (DMEM) and fetal bovine serum (FBS) were obtained from (Thermo Fisher Scientific Inc., Waltham, MA, USA).

Cell lines and cell culture. The human HCC MHCC-97L cell line used in the present study was purchased from the Cancer Research Institute of Zhongshan Hospital of Fudan University (Shanghai, China). The cells were cultured in high-glucose DMEM supplemented with heat-inactivated $10 \%$ fetal bovine serum, 100 units $/ \mathrm{ml}$ penicillin and $100 \mu \mathrm{g} / \mathrm{ml}$ streptomycin at $37^{\circ} \mathrm{C}$ in a humidified incubator containing a $5 \% \mathrm{CO}_{2}$ atmosphere.

Tissue array by in situ hybridization (ISH). The expression profile of miRNA-34a-5p was examined. A total of 180 oligonucleotide DNA probes were used for the detection of human
Table I. Expression level of miRNA-34a-5p in human Hepatocellular carcinoma tissue chip.

\begin{tabular}{lll}
\hline Expression of miRNA-34a-5p & Staining score & P-value \\
\hline Tumor & $4.28(1.46-7.10)$ & $<0.001$ \\
Pericarcinomatous & $7.23(4.40-10.06)$ & \\
\hline
\end{tabular}

miRNA-34a-5p. Each sample was independently hybridized to one array. Hybridization signals were detected using a DNA chip image analyzer (dChip software, http://www. hsph.harvard.edu/cli/complab/dchip/). The hybridized signal intensities were normalized to the intensities of synthetic oligonucleotide DNA probes. Finally, the normalized data from each sample were graded by expression levels relative to the median.

Semi-quantitative assessment. The expression level of miRNA-34a-5p was tested by integrating the percentage of stained cancer cells and the intensity of the staining. The intensity of staining was scored as follows: 0 , no staining; 1 , weak staining; 2 , moderate staining; and 3 , strong staining. The extent of the staining was scored according to the percentage of stained cells in the field of view, as follows: 0 , no staining; $1,0-25 \%$ stained cells; $2,26-50 \%$ stained cells; $3,51-75 \%$ stained cells; and $4,76-100 \%$ stained cells. The intensity score multiplied by the extent score was considered to be the overall ISH score. The ISH staining level was assessed and scored by two independent pathologists that were blinded to the clinicopathological and follow-up data of the samples.

Cell transfection. The miRNA-34a-5p mimics (5'-UGGCAGU GUCUUAGCUGGUUGU-3'), miRNA-34a-5p inhibitor (5'-ACAACCAGCUAAGACACUGCCA-3') and negative control miRNA (NC; 5'-CAGUACUUUUGUGUAGUA CAA-3') were synthesized by Guangzhou RiboBio, Co., Ltd. (Guangzhou, Guangdong, China). miRNA-34a-5p was overexpressed in MHCC-97L cells using miRNA-34a-5p mimics. The expression of miRNA-34a-5p was knocked down in MHCC-97L cells using miRNA-34a-5p inhibitors. The cells were seeded onto a six-well plate at a density of $2.5 \times 10^{5}$ cells/well $24 \mathrm{~h}$ prior to transfection. The cells were then transfected with $20 \mathrm{nM}$ miRNA-34a-5p mimics or $40 \mathrm{nM}$ inhibitors using Lipofectamine RNAiMax (Invitrogen) according to the manufacturer's instructions. The cells were harvested for further analysis $48 \mathrm{~h}$ subsequent to transfection. The small interfering (si)RNA for AXL (siAXL) was synthesized by Shanghai GenePharma Co., Ltd. (Shanghai, China), according to the two designed pairs of siAXL1 and siAXL2 siRNA, and the transfection was performed according to the manufacturer's instructions. Three independent experiments were performed.

Cell proliferation assay. Cell proliferation assays were performed using Cell Counting Assay. The MHCC-97L cells were seeded onto six-well plates in triplicate at a density of $\sim 3 \times 10^{5}$ cells/well and cultured in the growth medium. 
Table II. Multivariate Cox proportional-hazards analysis in the overall HCC patients.

\begin{tabular}{|c|c|c|c|}
\hline \multirow[b]{2}{*}{ Variable } & \multicolumn{3}{|c|}{ Overall survival } \\
\hline & HR & $95 \% \mathrm{CI}$ & P-value \\
\hline \multicolumn{4}{|l|}{ Age } \\
\hline$<50$ years vs. $\geq 50$ years & 1.355 & $0.810-2.266$ & 0.248 \\
\hline \multicolumn{4}{|l|}{ Gender } \\
\hline Male vs. female & 0.914 & $0.351-2.380$ & 0.853 \\
\hline \multicolumn{4}{|l|}{ Tumor size } \\
\hline$<5 \mathrm{~cm} \mathrm{vs.} \geq 5 \mathrm{~cm}$ & 1.342 & $0.767-2.349$ & 0.302 \\
\hline \multicolumn{4}{|l|}{ Tumor multiplicity } \\
\hline Single vs. mutiple & 2.514 & $0.981-6.443$ & 0.055 \\
\hline \multicolumn{4}{|l|}{ Histological grade } \\
\hline Poor vs. moderate and well & 1.029 & $0.798-1.328$ & 0.824 \\
\hline \multicolumn{4}{|l|}{ Stage } \\
\hline I + II vs. III + IV & 0.990 & $0.566-1.730$ & 0.971 \\
\hline \multicolumn{4}{|l|}{ HBV infection } \\
\hline Negative vs. positive & 1.013 & $0.400-2.567$ & 0.979 \\
\hline \multicolumn{4}{|l|}{ Liver cirrhosis } \\
\hline Negative vs. positive & 1.070 & $0.588-1.949$ & 0.824 \\
\hline \multicolumn{4}{|l|}{ Vascular invasion } \\
\hline Negative vs. positive & 1.556 & $0.685-3.536$ & 0.291 \\
\hline \multicolumn{4}{|l|}{ Serum AFP } \\
\hline $\begin{array}{l}\quad<20 \mu \mathrm{g} / 1 \mathrm{vs} . \geq 20 \mu \mathrm{g} / 1 \\
\text { miRNA-34a-5p expression }\end{array}$ & 2.841 & $1.517-5.322$ & 0.001 \\
\hline Low vs. high & 0.551 & $0.314-0.968$ & 0.038 \\
\hline
\end{tabular}

HCC, hepatocellular carcinoma; HR, hazard ratio; CI, confidence interval; HBV, hepatitis B virus; AFP, $\alpha$-fetoprotein.

Subsequent to 24,48 and $72 \mathrm{~h}$ of culturing, the numbers of cells per well were measured by counting the total cells using a hemocytometer and a microscope (DMI 4000B; Leica Microsystems GmbH, Wetzlar, Germany).

Apoptosis assay. The cells were plated into six-well plates at a density of $3 \times 10^{5}$ cells/well, and were transfected with miRNA-34a-5p mimics or miRNA-34a-5p inhibitors using Lipofectamine RNAiMax. The MHCC-97L cells were treated with cisplatin at a final concentration of $5 \mu \mathrm{g} / \mathrm{ml}$, and $12 \mathrm{~h}$ after the administration of cisplatin the cells were collected and stained with Annexin V-FITC and PI (cat. no. KGA108; Nanjing KeyGen Biotech Co., Ltd., Nanjing, Jiangsu, China). Flow cytometry was then performed to detect the apoptosis level of the transfected cells.

Luciferase reporter assay. For assays involving the full-length AXL 3'-untranslated region, the MHCC-97L cells were seeded at a density of 10,000 cells per well in a 96-well culture plate (Corning, Inc., Corning, NY, USA) and incubated for $24 \mathrm{~h}$ prior to transfection. In total, $1 \mu \mathrm{g}$ of the expression vector was cotransfected with negative control siRNA (siNC) or the human (h-)miRNA-34a-5p mimic (5'-UGGCAGUGUCUU AGCUGGUUGU-3') at a final concentration of $10 \mathrm{nM}$ using Lipofectamine RNAiMax. The expression levels of firefly and
Renilla luciferase were each measured 24-h post-transfection on a modulus microplate reader using the Luciferase Assay system (GeneCopoeia, Rockville, MD, USA). To generate the wild-type and mutant seed sequence expression constructs, the expression of Renilla and firefly luciferase was measured $48 \mathrm{~h}$ post-transfection in the MHCC-97L cells, with 25,000 cells per well, using the Dual-Luciferase Reporter Assay System (Promega, Madison, WI, USA). The mean \pm standard error of the mean is reported for each transfection condition $(n=3)$. The statistical significance of differences between the groups was determined by a two-tailed unpaired $t$-test for the means of the cells transfected with siNC, wild-type mimic and mutant mimic constructs.

Western blot analysis. Western blot analysis was performed to assess AXL and $\beta$-actin expression. Protein extracts from cultured cells were prepared by suspending cells in lysis buffer containing $0.01 \%$ EDTA, $0.1 \%$ Triton X-100 and $10 \%$ proteinase inhibitor. Protein concentrations were quantified using a protein assay kit (KGPBCA; Nanjing KeyGen Biotech Co., Ltd.). Briefly, $50 \mathrm{mg}$ of lysates were separated on $12 \%$ SDS-PAGE and transferred to polyvinylidene difluoride membranes. The membranes were probed overnight at $4^{\circ} \mathrm{C}$ with primary antibodies against human AXL (dilution, $1: 1,000)$ and $\beta$-actin (dilution, 1:1,000), followed by incubation with secondary antibodies (Cell Signaling Technology, Inc.) at a 1:5,000 dilution for $1 \mathrm{~h}$. The signal was visualized using enhanced chemiluminescence.

Statistical analysis. All assays were performed in triplicate. The data are expressed as the mean \pm standard deviation. The statistical analyses were performed using Student's $t$-test. $\mathrm{P}<0.05$ was considered to indicate a statistically significant difference. Statistical analyses were performed using SPSS software, version 20.0 (IBM, Armonk, NY, USA).

\section{Results}

The expression of miRNA-34a-5p in HCC tissues was significantly decreased compared with the pericarcinomatous areas of the tissue chips. To investigate the expression of miRNA-34a-5p in hepatocellular carcinoma, the tissue chips consisted of pairs of malignant and pericarcinomatous tissues that were derived from tumor samples of 114 patients with HCC that underwent radical surgery. An ISH assay was conducted to test the levels of miRNA-34a-5p in the tissue chip. The expression of miRNA-34a-5p in hepatocellular carcinoma tissues was reduced compared with peritumorial tissues $(\mathrm{P}<0.01$; Fig. 1A and $\mathrm{B})$, which was illustrated by the finding that the average staining scores for tumor and pericarcinomatous tissues were 4.28 and 7.23 , respectively $(\mathrm{P}<0.001$; Table I).

High expression of miRNA-34a-5p was associated with a favorable OS time in patients with HCC. miRNA-34a-5p demonstrated a heterogeneous expression level in HCC tissues (Fig. 1C). In order to identify the association between miRNA-34a-5p and the clinical outcome, the expression level of miRNA-34a-5p was dichotomized into two categories: the cutoff value of the receiver operating characteristic curve were used to separate the relative 
A

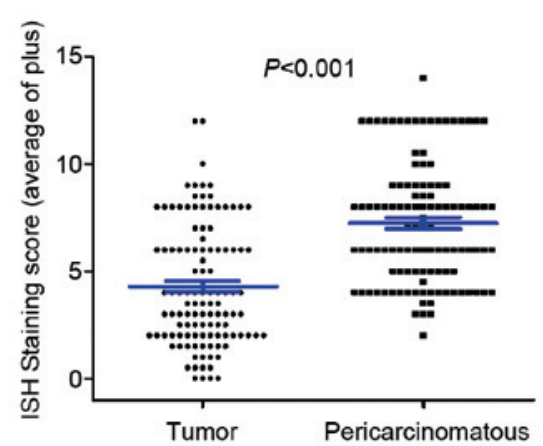

C

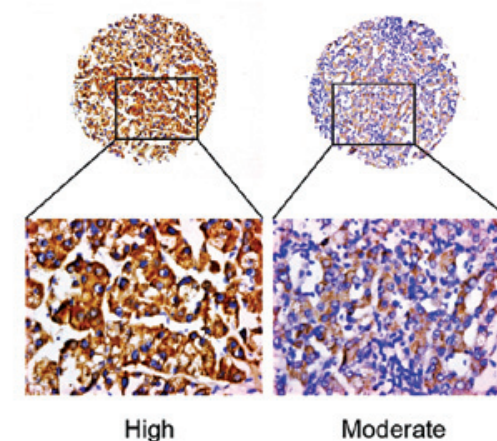

D

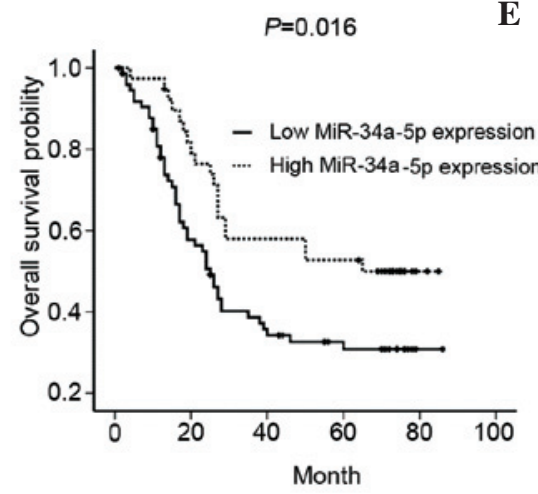

B
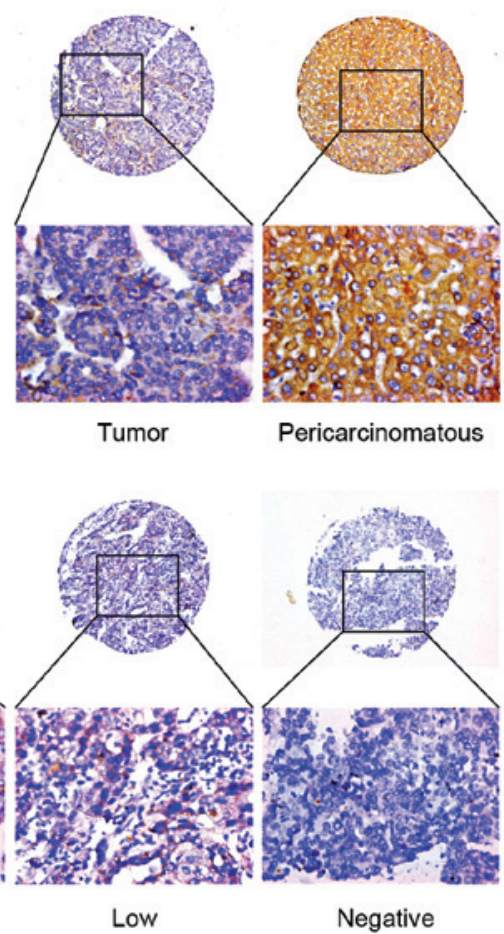

$\mathbf{E}$

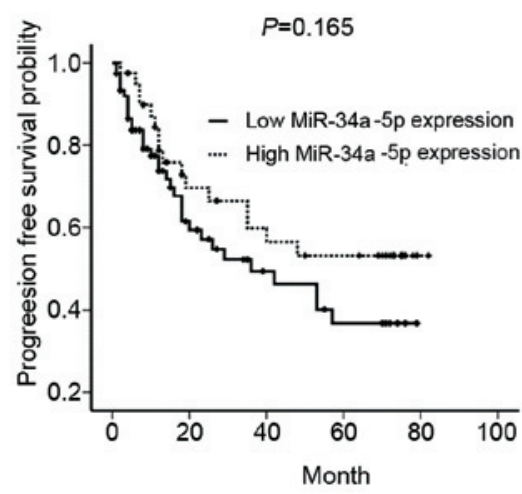

Figure 1. Expression of miRNA-34a-5p in the microarray and survival analysis of HCC tissues. (A) The scatter diagram represents the expression of miRNA-34a-5p in tumor and pericarcinomatous tissues, respectively, which was significantly different between the two tissue types $(\mathrm{P}<0.001)$. (B) In situ hybridization was performed to detect the expression of miRNA-34a-5p in HCC and pericarcinomatous tissues. (C) In situ hybridization was performed to verify the different degree of miRNA-34a-5p expression in HCC tissues. (D and E) Kaplan-Meier survival analysis was performed to assess the effect of miRNA-34a-5p on the overall survival and progression-free survival times of patients with HCC. HCC, hepatocellular carcinoma; miRNA-34a-5p, microRNA-34a-5p; MiR-34a, microRNA-34a.

expression levels of miRNA-34a-5p into low expresshigher and lower expression groups. Subsequently, Kaplan-Meier analysis revealed that lower expression of miRNA-34a-5p significantly decreased the OS time $(\mathrm{P}=0.016$; Fig. 1D) without influencing the PFS time $(\mathrm{P}=0.165$; Fig. $1 \mathrm{~F})$ in $\mathrm{HCC}$ patients.

Furthermore, multivariate Cox regression analysis demonstrated that the expression levels of serum $\alpha$-fetoprotein $[\mathrm{P}=0.01$; hazard ratio $(\mathrm{HR}), 2.841]$ and miRNA-34a-5p $(\mathrm{P}=0.038 ; \mathrm{HR}, 0.551)$ were independent prognostic biomarkers for the prediction of OS time. Other characteristics, consisting of age, gender, tumor size, tumor multiplicity, histological grades, stage, liver cirrhosis, HBV infection and vascular invasion, were not significantly associated with OS time (Table II).

miRNA-34a-5p inhibited proliferation of the HCC cell line. In order to identify the role of miRNA-34a-5p in the proliferation of HCC cells, miRNA-34a-5p mimics were transfected into in the human MHCC-97L cell line. Cell counting illustrated that cell proliferation was inhibited by forced expression of miRNA-34a-5p, with the best inhibition effect being demonstrated at $48 \mathrm{~h}$ subsequent to transfection $(\mathrm{P}<0.05$; Fig. $2 \mathrm{~A})$. In addition, the miRNA-34a-5p inhibitor was transfected into in the human MHCC-97L cell line and it was found that cell proliferation was significantly promoted $(\mathrm{P}<0.05$; Fig. $2 \mathrm{~B})$.

miRNA-34a-5p elevated cell apoptosis and decreased the chemoresistance of HCC cells to cisplatin. Flow cytometry was utilized to assess the effects of miRNA-34a-5p on cell apoptosis in the MHCC-97L cell line. Transfection of miRNA-34a-5p mimics into the MHCC-97L cell line significantly increased cell apoptosis by at least two-fold $(\mathrm{P}<0.05$; Fig. 2C). However, transfection of miRNA-34a-5p inhibitors did not reduce the 
A

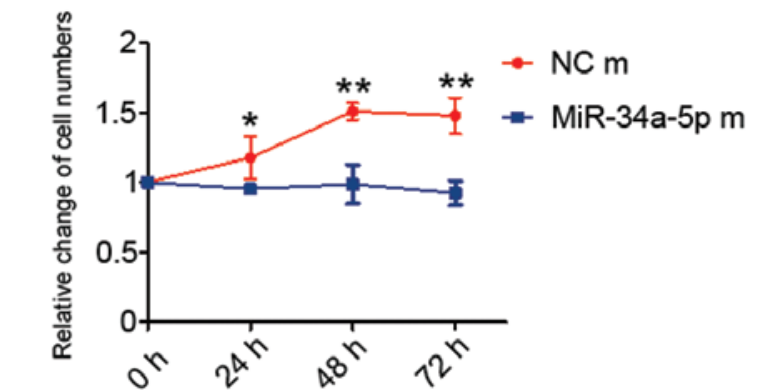

B

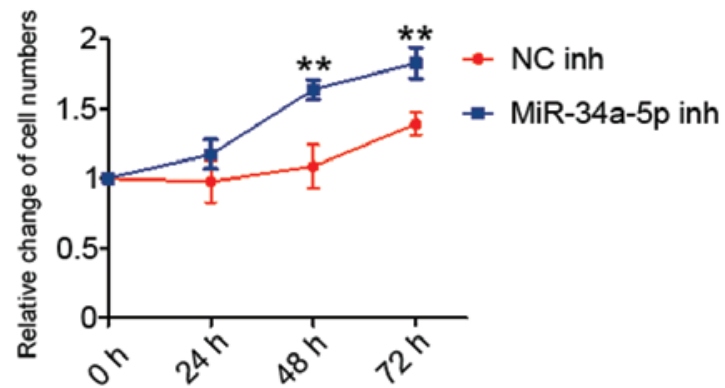

C

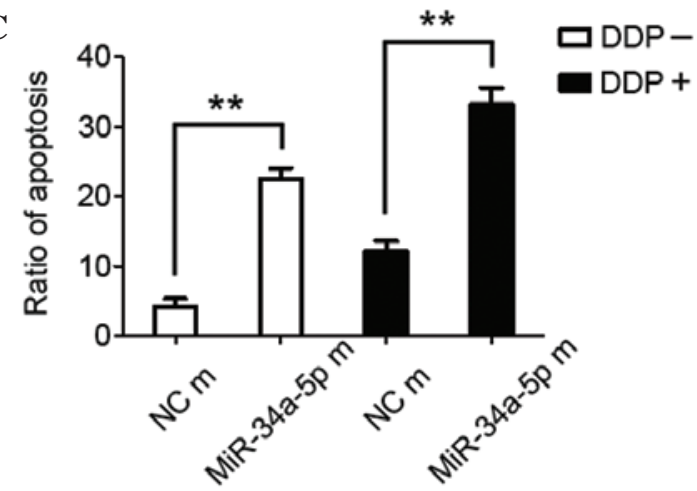

D

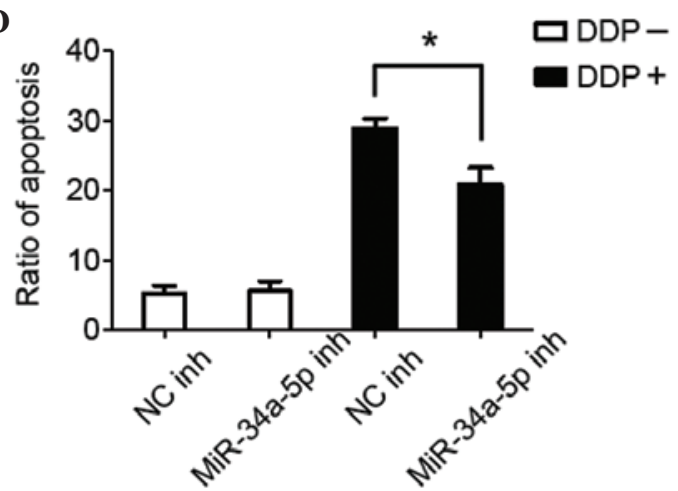

$\mathbf{F}$

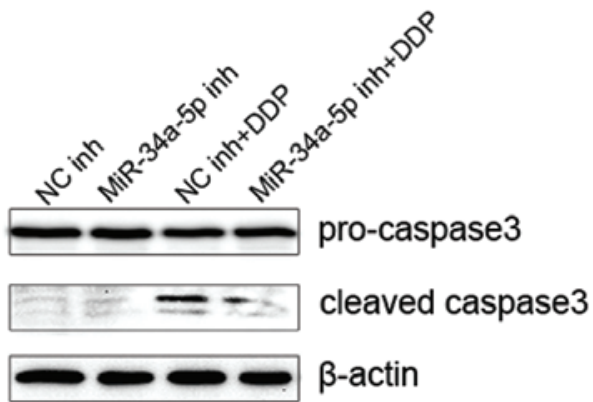

Figure 2. Forced expression of miRNA-34a-5p inhibits proliferation and promotes cell apoptosis. (A) Relative changes in the numbers of 97L cells transfected with the NC or miRNA-34a-5p mimic $(\mathrm{P}<0.05)$. (B) Relative changes in the number of $97 \mathrm{~L}$ cells transfected with the NC or miRNA-34a-5p inhibitor $(\mathrm{P}<0.05)$ (C and D) Flow cytometry was performed to detect the apoptosis ratio in 97L cells transfected with miRNA-34a-5p mimics or inhibitors, with or without pretreatment with cisplatin $(\mathrm{P}<0.05)$. ( E and F) Western blot analysis was performed to detect the protein expression of cleaved caspase-3 in the $97 \mathrm{~L}$ cells transfected with miRNA-34a-5p mimics or inhibitors, with or without pre-treatment with cisplatin $(\mathrm{P}<0.05)$. All experiments were performed in triplicate. ${ }^{*} \mathrm{P}<0.05$ and ${ }^{* *} \mathrm{P}<0.001$. miRNA-34a-5p, microRNA-34a-5p; MiR-34a, microRNA-34a; NC m, negative control mimic; NC inh, NC inhibitor; MiR-34a m, MiR-34a mimic; MiR-34a inh, MiR-34a inhibitor; DDP, cisplatin.

cell apoptosis ratio, which was quite low. To further validate the aforementioned results, cleaved caspase- 3 was tested by western blot analysis in the same setting. It was found that the expression of cleaved caspase- 3 was increased subsequent to transfection with miRNA-34a-5p mimics ( $\mathrm{P}<0.05$; Fig. 2E). Consistent with the results of flow cytometry, inhibition of miRNA-34a-5p in the MHCC-97L cells did not reduce the expression level of cleaved caspase-3, which was evidently low (P<0.05; Fig. 2F).

Cisplatin/DPP treatment enhanced apoptosis in the MHCC-97L cells. Therefore, cisplatin-treated MHCC-97L cells were used to determine the influence of miRNA-34a-5p on the chemoresistance of HCC cells. Firstly, transfection of miRNA-34a-5p mimics into the cisplatin-treated MHCC-97L cells significantly increased the level of apoptosis $(\mathrm{P}<0.05$; Fig. 2C). The apoptosis ratio of MHCC-97L cells administered with cisplatin was consistently reduced through inhibition of miRNA-34a-5p ( $<<0.05$; Fig. 2D). The findings of the western blot analysis further confirmed these results. The levels of the apoptosis protein activated caspase-3 were elevated by treatment with cisplatin. This elevation was enhanced by transfecting cells with miRNA-34a-5p mimics. By contrast, the levels of cleaved caspase-3 were decreased by transfection with the miRNA-34a-5p inhibitor $(\mathrm{P}<0.05$; Fig. $2 \mathrm{D}$ and $\mathrm{F})$.

AXL was the direct target of miRNA-34a-5p. In order to identify the specific target gene of miRNA-34a-5p that mediates the promotion of the apoptosis ratio, the Targetscan (Whitehead Institute for Biomedical Research, Cambridge, MA, USA) and MiRbase (Griffiths-Jones Lab, University of Manchester, Manchester, UK) databases were consulted and searched for the relative gene of proliferation. Among the associated target genes from the initial search were the AXL, PTPN18, CDKN1C and BAK genes, which were considered to be the most likely 
A

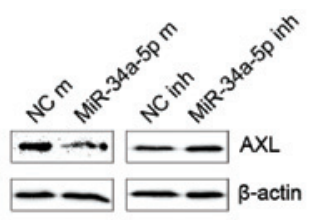

C

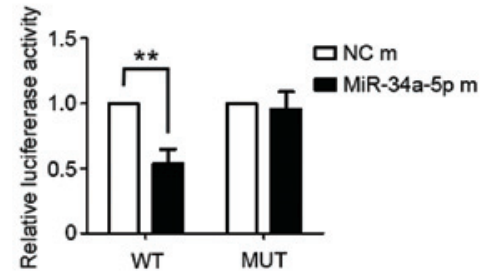

$\mathbf{F}$

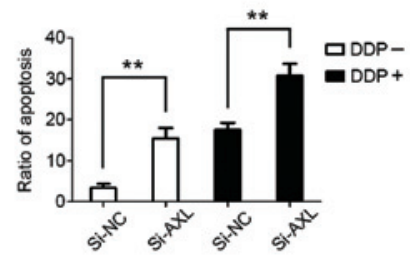

B
WT 3' UTR 5' GAUCCAAGCUAAG-CACUGCCA 3'

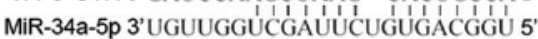
MUT 3' UTR 5' GAUCCAUCGAUUC-GUGACGGU3'

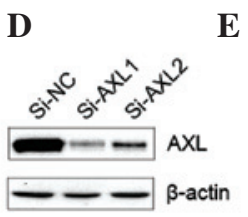

G
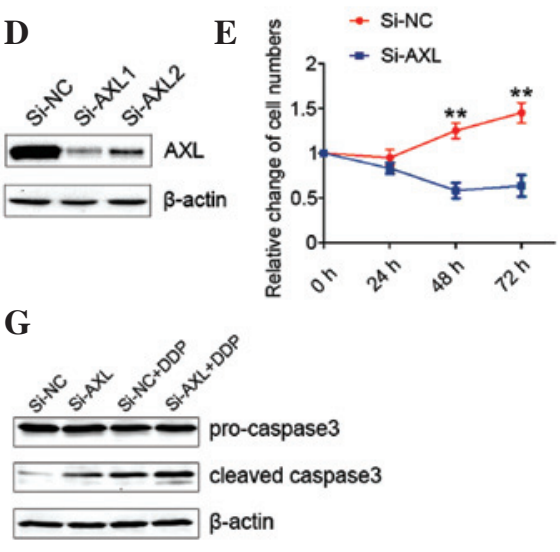

Figure 3. miRNA-34a-5p modulated the chemo-sensitivity by targeting AXL. (A) Western blot were performed to detected the expression of AXL in 97L cells transfected with miRNA-34a-5p mimics or inhibitors $(\mathrm{P}<0.05)$. (B) The binding situ of wild type and mutant AXL for miRNA-34a-5p. (C) Luciferase Reporter assay were performed to test the relative luciferase activity of wild type or mutant AXL after 97L cell were transfected with miRNA-34a-5p, respectively. (D) Western blot assay were conducted to detect the expression of AXL after 97L cells were transfected with siAXL (P<0.05). (E) Cell proliferation assays were performed to detect the relative changes of cell numbers in $97 \mathrm{~L}$ cells transfected with siNC or siAXL, respectively (P<0.05). (F) Flow cytometry assay were performed to detect the apoptosis ratio in 97L cells transfected with siAXL with or without pretreatment of cisplatin, respectively $(\mathrm{P}<0.05)$. (G) Western blot were performed to detect the expression of apoptosis protein caspase-3 in 97L cells transfected with siAXL with or without pretreatment of cisplatin, respectively $(\mathrm{P}<0.05)$. All experiments were performed in triplicate $\left({ }^{* *} \mathrm{P}<0.001\right)$. miRNA-34a-5p, microRNA-34a-5p; Si-NC, negative control small interfering RNA; Si-AXL, AXL small interefering RNA; WT, wild-type; MUT, mutant.

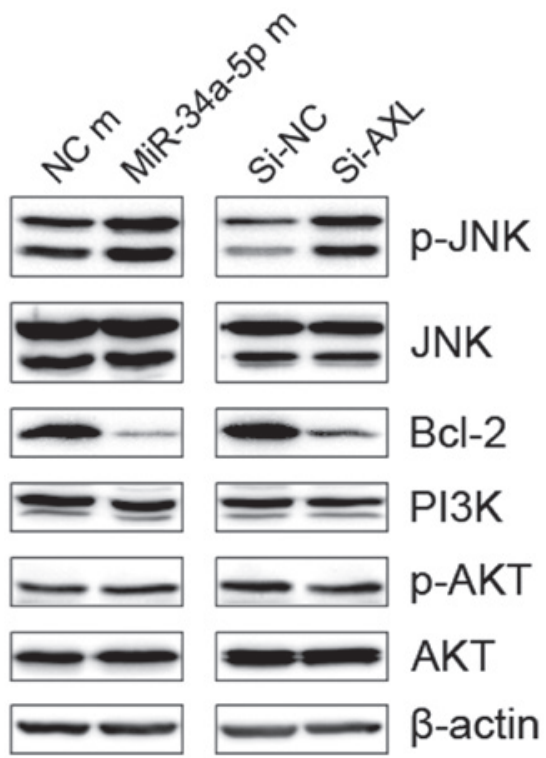

Figure 4. miRNA-34a-5p may modulate the chemosensitivity of HCC cells through the JNK-Bcl-2 signaling pathway. Western blot analysis was performed to detect the expression of JNK, AKT, PI3K and Bcl-2, as well as the phosphorylation of JNK and AKT in HCC cells. NC, negative control; miRNA-34a-5p, microRNA-34a-5p; miR-34a, miRNA-34a; Si-NC, small interfering NC RNA; Si-AXL, small interfering RNA for AXL; JNK. c-Jun N-terminal kinase; p-JNK, phosphorylated JNK; Bcl-2; B-cell lymphoma 2; PI3K, phosphoinositide 3-kinase; p-AKT, phosphorylated AKT; HCC, hepatocellular carcinoma.

target genes involved in the miRNA-34a-5p-induced decrease in cell proliferation and increase in apoptosis. Western blot analysis revealed that the expression levels of PTPN18,
CDKN1C and BAK were not downregulated by transfection with miRNA-34a-5p mimics.

The expression of AXL was found to be significantly reduced in the cells that were transfected with miRNA-34a-5p mimics, while enhanced AXL expression was observed in the cells that were treated with miRNA-34a-5p inhibitors $(\mathrm{P}<0.05$; Fig. 3A). This potential direct interaction between miRNA-34a-5p and AXL was further investigated by the binding in situ analysis in the Targetscan, which revealed that miRNA-34a-5p directly bound with wild-type AXL and lost this capability when AXL was mutated (Fig. 3B). The luciferase reporter assay was then utilized to confirm this direct interaction. The primers for mutant and wild-type AXL were designed and $1 \mu \mathrm{g}$ each of the expression vector and h-miRNA-34a-5p mimic were co-transfected into the MHCC-97L cells. The luciferase reporter assay illustrated that the luciferase activity of wild-type AXL was decreased significantly when co-transfected with h-miRNA-34a-5p mimics. The luciferase activity of mutant AXL was also similar to that of the control group $(\mathrm{P}<0.01$; Fig. 3C).

miRNA-34a-5p modulates cell proliferation, apoptosis and chemoresistance by targeting AXL. The influence of AXL on the proliferation and apoptosis of MHCC-97L cells was evaluated to determine the mediating role of AXL in the decreased cell proliferation and increased apoptosis induced by miRNA-34a-5p. siAXL was transfected into the MHCC-97L cell line and significantly reduced the expression of AXL $(\mathrm{P}<0.05$; Fig. 3D). Cell counting revealed that silencing AXL completely suppressed cell proliferation $(\mathrm{P}<0.05$; Fig. $3 \mathrm{E})$. In addition, transfection with siAXL increased the apoptosis ratio 
of MHCC-97L cells $(\mathrm{P}<0.05$; Fig. 3F). Notably, transfection of cells with siAXL enhances the apoptosis induced by cisplatin. The results of flow cytometry on cell apoptosis were further confirmed by western blot analysis of the expression of cleaved caspase-3 ( $\mathrm{P}<0.05$; Fig. 3G). These results were in accordance with the aforementioned biological behaviors induced by the ectopic expression of miRNA-34a-5p.

miRNA-34a-5p may act through the JNK-Bcl-2 signaling pathway. In order to reveal the signaling pathway by which miRNA-34a-5p exerted anticancer effects in HCC cells, western blot analysis was used to assess the alteration of several secondary messengers in various signaling pathways. The present results revealed that transfection with miRNA-34a-5p mimics significantly increased the phosphorylation of JNK and decreased the levels of Bcl-2. In addition, transfection with miRNA-34a-5p mimics demonstrated little effect on the expression levels of PI3K and p-AKT ( $\mathrm{P}<0.05$; Fig. 4). In addition, silencing of AXL by transfection of cells with siAXL also increased the levels of p-JNK and decreased the expression of $\mathrm{Bcl}-2$, without influencing the level of PI3K and p-AKT. All these data indicate that miRNA-34a-5p may exert its anticancer function by targeting AXL through the p-JNK-Bcl-2 signaling pathway.

\section{Discussion}

An increasing quantity of evidence suggests that miRNA-34a is a key mediator of p53 tumor suppression (11) that plays an important role in the development of HCC. miRNA-34a demonstrates anticancer properties through its action on the proliferation, apoptosis, invasion and metastasis of $\mathrm{HCC}$ cells (5-8). However, no studies have investigated the role of miRNA-34a in chemoresistance, which is a critical feature of HCC and has led to the limited survival of patients with advanced disease. The present study initially identified the role of miRNA-34a-5p in the chemoresistance of HCC cells. miRNA-34a-5p was found to significantly reduce the chemoresistance of HCC cells, as apoptosis induced by cisplatin was increased by transfection of cells with miRNA-34a-5p mimics. In addition, miRNA-34a has been reported to sensitize HCC cells to sorafenib (10). The function of miRNA-34a-5p in facilitating systematic therapy was confirmed at a clinical level in the present study. Patients with various miRNA-34a expression levels demonstrated similar PFS times, but different OS times, which indicated that the impact of miRNA-34a on the prognosis of HCC patients was mainly exerted subsequent to the recurrence of the malignancy. Transcatheter arterial chemoembolization (TACE) and systematic therapy, which was mainly chemotherapy using sorafenib, were the main strategies for the treatment of advanced HCC (3). Thus, sensitivity to chemotherapy and sorafenib became a critical factor for the prognosis of patients with HCC, in which miRNA-34a evidently played an important role, as indicated by the present study and a previous study by Yang et al (10).

The present results were in accordance with the majority of the findings from previous studies. Previous studies did not report consistent findings on the function of miRNA-34a. Dang et al (9) reported results that were consistent with the present findings that miRNA-34a-5p decreased cell proliferation and increased apoptosis in three HCC cell lines, which differed from the present cell lines and consisted of the HepG2, HepB3 and SNU449 cell lines. However, Li et al (6) did not identify this function of miRNA-34a in HepG2 cells. In addition, Li et al (6), Dang et al (9) and the present study concluded that downregulation of the expression of miRNA-34a was significantly increased in HCC tissues. However, Pineau et al (12) observed contrasting results. The different sources of the samples and the various assays performed may partly explain the discordance of the varying results.

In the present study, the miRNA-34a/AXL pathway was identified as a novel pathway leading to the chemoresistance of HCC. AXL is a member of the Tyro3, AXL and MER receptor tyrosine kinase family and it plays a diverse role in multiple cellular processes, including survival, apoptosis and proliferation (13), with the exception of chemoresistance. In addition, previous studies on the function of AXL in HCC were mainly focused on the oncogenesis, metastasis and proliferation of the cells (14-18). In the present study, it was found that transfection of HCC cells with siAXL enhanced the apoptosis induced by cisplatin in HCC cells. The interaction between miRNA-34a and AXL was initially identified in breast cancer by Mackiewicz et al (19). Therefore, the miRNA-34a-AXL pathway may play a significant role in HCC patients with a low expression of miRNA-34a.

In the current study, it was found that the miRNA-34a-AXL pathway mediated the phosphorylation of JNK and expression of Bcl-2, which are each apoptosis-associated messengers (20). Thus, the apoptosis effect induced by the miRNA-34a-AXL pathway in HCC may be mediated by JNK and Bcl-2. However, the roles of JNK and Bcl-2 in this biological process have yet to be fully identified.

In conclusion, the present study identified that miRNA-34a-5p enhanced the sensitivity of chemotherapy by targeting AXL in hepatocellular carcinoma. Low expression of miRNA-34a-5p in HCC tissues yielded an unfavorable OS time for patients with HCC that received radical surgery by promoting proliferation and chemoresistance in HCC cells.

\section{Acknowledgements}

This study was supported by grants from the National Natural Science Foundation of China (grant no. 81372374), the Combination Project of Production, Education and Research from Guangdong Province and Ministry of Education (grant no. 2012B091100460), and the Science and Technology Planning Project of Guangdong Province (grant nos. 2011B031800076, 2011B031800014 and 2012B031800259).

\section{References}

1. Jia CC, Wang TT, Liu W, et al: Cancer-associated fibroblasts from hepatocellular carcinoma promote malignant cell proliferation by HGF secretion. PLoS One 8: e63243, 2013.

2. Li X, Dong M, Lin Q, et al: Comparison of current staging systems for advanced hepatocellular carcinoma not amendable to locoregional therapy as inclusion criteria for clinical trials. Asia Pac J Clin Oncol 9: 86-92, 2013.

3. Graf D, Vallböhmer D, Knoefel WT, et al: Multimodal treatment of hepatocellular carcinoma. Eur J Intern Med 25: 430-437, 2014. 
4. Sheng L, Xiong M,Li C and Meng X: Reversing multidrug-resistant by RNA interference through silencing MDR1 gene in human hepatocellular carcinoma cells subline Bel-7402/ADM. Pathol Oncol Res 20: 541-548, 2014.

5. Cheng J, Zhou L, Xie QF, et al: The impact of miR-34a on protein output in hepatocellular carcinoma HepG2 cells. Proteomics 10 $1557-1572,2010$

6. Li N, Fu H, Tie Y, et al: miR-34a inhibits migration and invasion by down-regulation of c-Met expression in human hepatocellular carcinoma cells. Cancer Lett 275: 44-53, 2009.

7. Tryndyak VP, Ross SA, Beland FA and Pogribny IP Down-regulation of the microRNAs miR-34a, miR-127 and miR-200b in rat liver during hepatocarcinogenesis induced by a methyl-deficient diet. Mol Carcinog 48: 479-487, 2009.

8. Yang P, Li QJ, Feng Y, et al: TGF- $\beta$-miR-34a-CCL22 signaling-induced Treg cell recruitment promotes venous metastases of HBV-positive hepatocellular carcinoma. Cancer Cell 22: 291-303, 2012.

9. Dang Y, Luo D, Rong M and Chen G: Underexpression of miR-34a in hepatocellular carcinoma and its contribution towards enhancement of proliferating inhibitory effects of agents targeting c-MET. PLoS One 8: e61054, 2013.

10. Yang F, Li QJ, Gong ZB, et al: MicroRNA-34a targets Bcl-2 and sensitizes human hepatocellular carcinoma cells to sorafenib treatment. Technol Cancer Res Treat 13: 77-86, 2014.

11. Bommer GT, Gerin I, Feng Y, et al: p53-mediated activation of miRNA34 candidate tumor-suppressor genes. Curr Biol 17: 1298-1307, 2007.

12. Pineau P, Volinia S, McJunkin K, et al: miR-221 overexpression contributes to liver tumorigenesis. Proc Natl Acad Sci USA 107: 264-269, 2010
13. Linger RM, Keating AK, Earp HS and Graham DK: TAM receptor tyrosine kinases: biologic functions, signaling, and potential therapeutic targeting in human cancer. Adv Cancer Res 100: 35-83, 2008.

14. He L, Zhang J, Jiang L, et al: Differential expression of Axl in hepatocellular carcinoma and correlation with tumor lymphatic metastasis. Mol Carcinog 49: 882-891, 2010.

15. Lee HJ, Jeng YM, Chen YL, Chung L and Yuan RH: Gas6/Axl pathway promotes tumor invasion through the transcriptional activation of Slug in hepatocellular carcinoma. Carcinogenesis 35: 769-775, 2014.

16. Li J, Jia L, Ma ZH, Ma QH, Yang XH and Zhao YF: Axl glycosylation mediates tumor cell proliferation, invasion and lymphatic metastasis in murine hepatocellular carcinoma. World J Gastroenterol 18: 5369-5376, 2012.

17. Xu J, Jia L, Ma H, Li Y, Ma Z and Zhao Y: Axl gene knockdown inhibits the metastasis properties of hepatocellular carcinoma via PI3K/Akt-PAK1 signal pathway. Tumour Biol 35: 3809-3817, 2014.

18. Xu MZ, Chan SW, Liu AM, et al: AXL receptor kinase is a mediator of YAP-dependent oncogenic functions in hepatocellular carcinoma. Oncogene 30: 1229-1240, 2011.

19. Mackiewicz M, Huppi K, Pitt JJ, Dorsey TH, Ambs S and Caplen NJ: Identification of the receptor tyrosine kinase AXL in breast cancer as a target for the human miR-34a microRNA. Breast Cancer Res Treat 130: 663-679, 2011.

20. Liu J and Lin A: Role of JNK activation in apoptosis: A double-edged sword. Cell Res 15: 36-42, 2005. 